\title{
The joint meeting of 54th Annual Congress of the Japanese Society of Pediatric Surgeons and 7th International Sendai Symposium on Biliary Atresia in Sendai Japan
}

\author{
Masaki Nio $^{1} \cdot$ Hiroaki Kitagawa ${ }^{2}$
}

Accepted: 5 September 2017 / Published online: 4 October 2017

(c) Springer-Verlag GmbH Germany 2017

The joint meeting of the 54th Japanese Society of Pediatric Surgeons (JSPS) and 7th International Sendai Symposium on Biliary Atresia (ISSBA) was held in Sendai, Japan, on 11-13th May 2017. The first ISSBA organized by Prof. Kasai was held in 1972.

The meeting was very well attended with the total number of 1137 participants, including a large number of overseas guests from many countries. It was a very successful meeting with 711 papers presented relating to clinical pediatric surgery and pediatric surgery research.

There were seven symposiums in ISSBA. ISSBA topics that included Registries and Guidelines, Etiology and Pathophysiology, Screening and Diagnosis, Asian Experiences,
Treatment and Perioperative Care, Long-Term Management and Late Complications and Long-term Outcome and Liver Transplantation. Key-note lectures were given by experts in their respective fields by 27 speakers from all over the word. Fifteen articles were selected for publication in this special issue of Pediatric Surgery International including many review article.

We, the guest editors of this special issue of Pediatric Surgery International, are honored to share the contents of this highly successful meeting in Sendai with all the pediatric surgeons around the world.
Masaki Nio

mnio@ped-surg.med.tohoku.ac.jp

Hiroaki Kitagawa

h2kita@marianna-u.ac.jp

Sedai, Japan

2 Kawasaki, Japan 